See discussions, stats, and author profiles for this publication at: https://www.researchgate.net/publication/324177043

\title{
Improved Outdoor Localization Based on Weighted Kullback-Leibler Divergence for Measurements Diagnosis
}

Article in IEEE Intelligent Transportation Systems Magazine · November 2018

DOI: $10.1109 / \mathrm{MITS} .2018 .2879165$

CITATIONS

3

2 authors:

A. Joelle Al Hage

Université de Technologie de Compiègne

23 PUBLICATIONS 126 CITATIONS

SEE PROFILE
READS

211

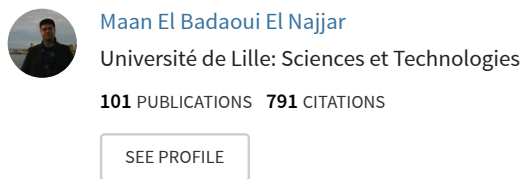

Some of the authors of this publication are also working on these related projects:

Personal Localization System View project

Multisensory Data Fusion with Detection and Exclusion of Default Based on Kullback-Leibler Divergence View project 


\title{
Improved Outdoor Localization Based on Weighted Kullback-Leibler Divergence for Measurements Diagnosis
}

\author{
Joelle Al Hage and Maan E.El Najjar
}

\begin{abstract}
The Global Navigation Satellite System (GNSS) is growing in usefulness for navigation in outdoor environment. However, the measurements from the satellites (pseudorange, Doppler) could be obscured or degraded due to different phenomena such as the multipath and interference. Therefore, in order to ensure the continuity and the integrity of localization, the fusion of these measurements with proprioceptive data is necessary. Adding also, the erroneous measurements should be detected and excluded from the fusion procedure.

In this work, we propose a tightly coupled architecture (GNSS/Odometer) with pseudorange Fault Detection and Exclusion (FDE) based on Weighted Kullback-Leibler Divergence (WKLD). Threshold optimization based on Kullback-Leibler Criterion $(K L C)$ is applied in order to replace classical approaches that fix heuristically the false alarm probability. The validation of this framework is studied on real experimental data using Septentrio receiver.
\end{abstract}

Index Terms-Localization, Weighted Kullback-Leibler Divergence, Fault Detection and Exclusion, threshold optimization, Tightly coupled GNSS/odometer

\section{INTRODUCTION}

$\mathbf{O}$ $\mathrm{NE}$ of the main challenge when navigating in outdoor environment is to ensure an accurate and reliable position estimation in presence of buildings and trees [1] [2]. In restricted sky view, the desirability of aiding the GNSS with proprioceptive data is relevant in order to ensure the continuity of localization. The proprioceptive sensors used in such data fusion refer to Inertial Measurement Unit (IMU), odometer and encoders [3]. Other methods rely on the use of exteroceptive sensors like the camera [4] and LiDAR [5].

For integrating GNSS with proprioceptive data, we distinguish loosely coupled and tightly coupled architectures [6][7][8][9]. The loosely coupled architecture consists in fusing the position estimated by the GNSS receiver with the dead reckoning estimation. However, tightly coupled uses a single filter to fuse GNSS and proprioceptive sensors measurements. The main advantage of the tightly coupled architecture is that the GNSS measurements could be used to update the dead reckoning estimation when less than four satellites are available. Many works highlight the benefits of tightly coupled architecture [10] in ensuring the integrity of localization in urban environment.

J. Al Hage is with Heudiasyc laboratory, UMR CNRS 7253, Université de technologie de Compiègne, 60200, Compiègne, France. M.E.El Najjar is with CRIStAL laboratory, UMR CNRS 9189, University of Lille, 59655 Villeneuve d'Ascq, France. E-mail: joelle.al-hage@hds.utc.fr.
Traditional Receiver Autonomous Integrity Monitoring (RAIM) methods are based only on the fault detection. However, recent GNSS receivers expand the RAIM to include an exclusion of faults ensuring the continuity of localization.

In general, in RAIM context, integrity monitoring is achieved by using:

- the Least Square Residual (LSR) [11]: The residual test is constructed from the difference between the measurements $(Z)$ and the estimations $(\hat{Z})$ obtained from the least square solution.

- the projection into the parity space [12]: The test variable is constructed in the parity space.

- comparison of pseudorange [13]: In the case that we have more than 4 visible satellites, a navigation solution is obtained by satisfying the first four measurements equations. The resulting solution is then used to predict the remaining pseudorange measurements. The difference between the predicted and the measured pseudorange is used to detect the presence of erroneous measurements.

- Multiple Solution Separation (MSS) [14] [15]: This method acts in the state space (position) which is not the case with the above mentioned methods. It is a more fundamental approach because in general we do not care about the error if it does not have a direct effect on the position. For the faults detection, a bank of filters should be used; for the fault exclusion another bank is added. This approach can be extended to the exclusion of multiple faults by adding, at each time, a new bank of filters. This extension has the disadvantage of a large computational load.

In urban environment, the probability of occurrence of multiple faults is quite high because of multipath and waves interference. The fault detection methods based on LSR, the projection into the parity space, and the comparison of pseudorange are mainly used for the detection of one fault at a time. Indeed, if the variable test exceeds the threshold value, we can't specify if the origin is one or more faults. In addition, a fault in one observation could have effect in another one due to satellites geometry and the correlation between the measurements.

In order to specify precisely the erroneous observations, several techniques are proposed in the literature and are based on two types of tests: Global Test (GT) and Local Tests (LT) [16] [17] [18]. The GT aims to detect the presence of faults and the LT intend to isolate the erroneous measurements by ana- 
lyzing standardized residuals $\left(w_{i}\right)$ obtained from the difference between the measured and the estimated pseudorange $Z_{i} . w_{i}$ are assumed to be normally distributed in the case of absence of defaults. Therefore, each $w_{i}$ that exceeds the threshold value $T$ corresponding to a probability $\left(1-\frac{P_{F}}{2}\right)$ of the Gaussian distribution is declared faulty, where $P_{F}$ is the false alarm probability. However, this strategy, using the recursive LT, can mark good measurements as erroneous. In order to mitigate this problem, several approaches are proposed in literature:

- Subset: If a measurements set is declared faulty using the GT, all possible combinations of measurements are checked in order to find a subset that excludes the faulty measurements. The subset that passes the GT and has the largest number of measurements is used for the position estimation. However, this method has a significant computational load.

- Forward-Backward: This method uses the GT and the LT. The first part of the algorithm, namely Forward, is applied to detect and exclude the erroneous measurements. If the GT indicates the presence of faults, the LT are applied in order to exclude the erroneous measurements. The Forward procedure is done in a recursive manner until no measurement is declared faulty. The exclusion of a healthy observation is possible due to the correlation between the measurements. Therefore, if $k$ measurements are excluded, the Backward procedure is applied by reintroducing iteratively the excluded measurements and by applying the GT. The set of measurements that passes this test is used for the position estimation.

- Danish method: This method is a re-weighted iterative least squares algorithm. It is very popular in geodetic applications. This method uses GT to verify the consistency of the measurements and LT to identify and reduce the weights of the outliers measurements.

In this paper, we propose an informational framework for localization in outdoor environment with a tightly coupled GNSS/Odometer. The tightly coupled architecture is chosen because of its attractive results in the urban environment where less than 4 satellites are in view. Adding also, this method is adapted for the diagnosis of the erroneous GNSS measurements.

The efficiency of the Information Filter (IF) [19] (the informational form of the Kalman Filter (KF)) has been proven for multi-sensor data fusion with FDE step [20]. Indeed, the update step of the IF is modeled as a simple summation of the information contributions of the different observations allowing a distributed data fusion and diagnosis architectures. Assimakis et al. [21] present the computational requirements of the Information and Kalman Filters. They demonstrated that the information filter is faster if the size of the observation vector is greater than $1.65 n$ for a time-varying system and than $0.75 n$ for a time-invariant system, with $n$ the size of the state vector. This can be verified by the fact that the informational filter is more advantageous in the correction step. In the prediction step, it is necessary to invert a matrix having the size of the state vector. By contrast, for the Kalman filter, the correction step requires the inversion of a matrix having the size of the observation vector.

In this work, we propose a FDE method of erroneous satellite measurements based on a bank of Extended IF (EIF). The method acts in the state space (position domain) since we are not interested by an error if it does not have a direct effect on the position estimation. Therefore, a fault is detected when the posteriori distribution (obtained from the update step of the EIF and using the pseudorange measurements) diverges from the priori distribution (obtained from the predicted step and using the encoders measurements). This divergence is quantified using the Weighted Kullback-Leibler Divergence $(W K L D)$. In the case of Gaussian distributions, the KullbackLeibler Divergence (KLD) [22], [23] takes into account the Mahalanobis distance (distance between the means of the data distributions) and the Burg matrix divergence between covariance matrices [24]. In this work, we propose to add a weight to each part of the KLD depending on the confidence that we have on them. This confidence is computed using the trace of the update covariance matrix. For the faults exclusion, a bank of EIF is generated $\left(E I F_{j}\right)$ in order to determine the information contribution of the pseudorange measurement $\left(\rho_{j}\right)$ and therefore, a set of residuals $\left(W K L_{j}\right)$ is constructed. The method is designed in a similar way as the Forward-Backward method so it will be able to exclude multiple faults.

In order to detect a fault, the $W K L D$ should be compared to a threshold value. In aeronautics domain, the RAIM algorithms are designed with a false alarm and miss detection probabilities which provide an agreement with the integrity risk standardized by ICAO (International Civil Aviation Organization) [25]. For urban applications, the conditions are different, therefore, the number of positions declared valid can be determined differently than the case of the aviation applications. In this work, we propose more elaborate method for the threshold calculation based on Kullback-Leibler Criterion $(K L C)$ [20]. It is based on the maximization of the information gain given by a decision on the true hypothesis (fault or non fault) $(K L C=\max (K L S))$. KLS (KullbackLeibler Summation) can be viewed as the summation of the information gain between the priori and the posteriori distributions given a particular value: $K L S=K L S_{0}+K L S_{1}$. The optimization using $K L S_{0}$ leads to a liberal threshold and that using $K L S_{1}$ leads to a conservative one [26]. The optimal threshold obtained from $K L C$ lies inside this interval and depends on the prior probability of default $\left(P_{0}\right)$ obtained using the historical system behavior and the Maximum Likelihood Estimation (MLE) [27].

The general approach of the method is given in figure 1

The main contributions of this paper are:

- Developing a tightly coupled (GNSS/odometer) with FDE approach able to deal with multiple faults,

- Using the information filter for state estimation and the Weighted Kullback-Leibler Divergence as a residual,

- Threshold optimization based on the Kullback-Leibler Criterion for GNSS applications.

This paper is organized as follows: section 2 presents the tightly coupled GNSS/Odometer based on the EIF. Section 3 details the FDE approach based on the $W K L D$. Threshold optimization based on the $K L C$ is shown in section 4 and it 


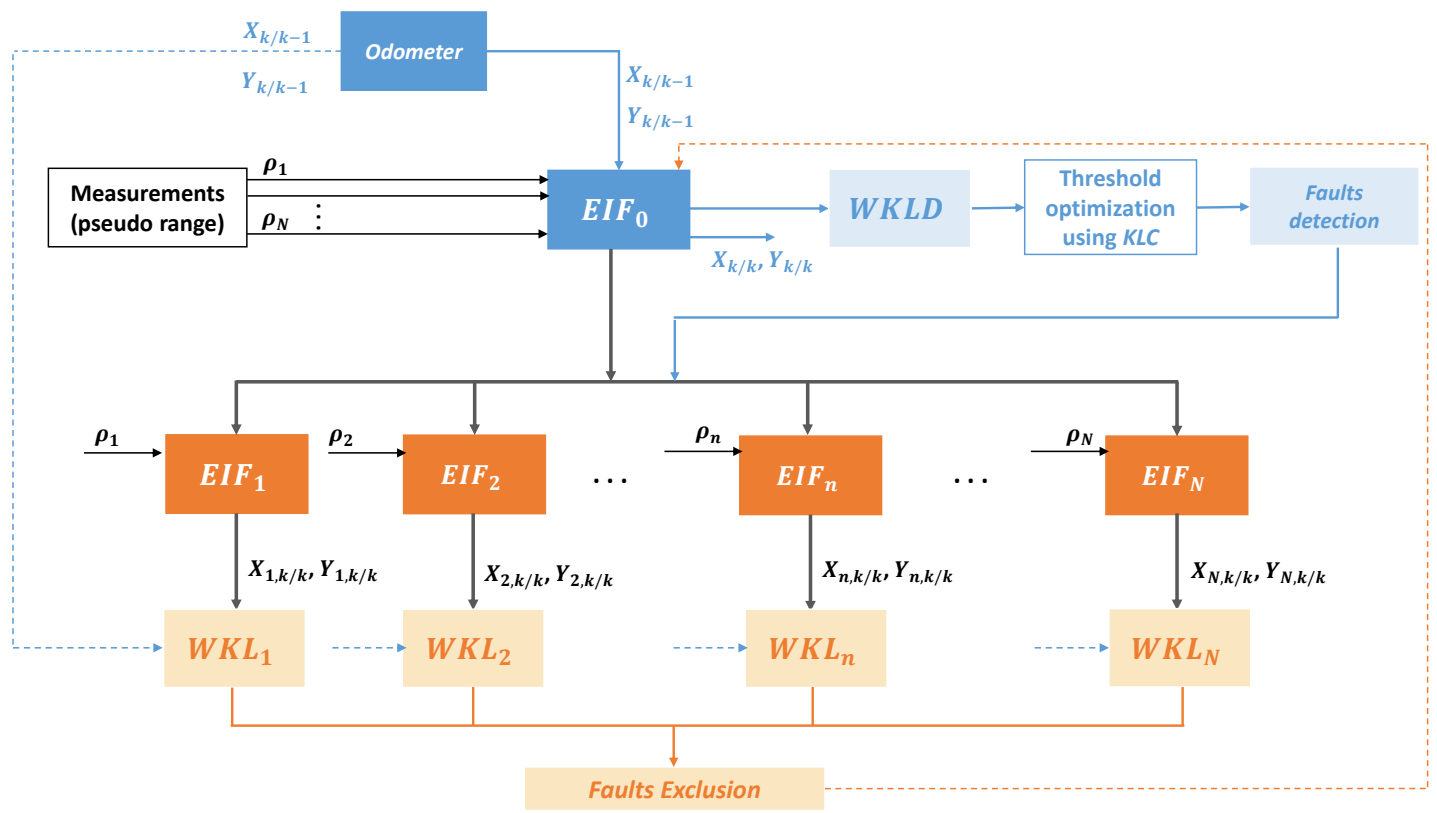

Fig. 1: The proposed approach for GNSS/Odometer data fusion with fault detection and exclusion.

is compared to other informational criteria. Results based on experimental data from a Septentrio receiver are shown and detailed in section 5 followed by a conclusion in section 6 .

\section{Tightly Coupled Architecture GNSS/Odometer}

In order to estimate the position of the vehicle through a tightly coupled architecture, we propose to use the EIF [19]. It is divided into two parts:

1) prediction where the state vector is estimated using the encoders measurements,

2) update where the estimation is corrected using the pseudorange measurements.

\section{A. Prediction step}

At instant $k$, the state vector is considered to be the 3D position of the vehicle in the Earth Centered Earth Fixed (ECEF), its orientation, the clock range $\left(c \delta t_{r, k}\right)$ and its variation:

$$
X_{k}=\left[\begin{array}{llllll}
x_{k} & y_{k} & z_{k} & \theta_{k} & c \delta t_{r, k} & c \frac{\partial \delta t_{r, k}}{\partial k}
\end{array}\right]^{T}
$$

$c$ is the speed of light.

$\delta t_{r, k}$ is the clock shift of the receiver at the moment of reception with respect to GPS reference time.

The propagation equation is obtained from the odometer model as follows:

$$
\begin{aligned}
X_{k+1 / k} & =T X_{k / k}+A_{k} u_{k}+w_{k} \\
& =f\left(X_{k / k}, u_{k}\right)+w_{k}
\end{aligned}
$$

where:

$T$ is the transition matrix defined by:

$$
T=\left(\begin{array}{cccccc}
1 & 0 & 0 & 0 & 0 & 0 \\
0 & 1 & 0 & 0 & 0 & 0 \\
0 & 0 & 1 & 0 & 0 & 0 \\
0 & 0 & 0 & 1 & 0 & 0 \\
0 & 0 & 0 & 0 & 1 & T_{e} \\
0 & 0 & 0 & 0 & 0 & 1
\end{array}\right)
$$

$T_{e} \quad$ is the sampling period.

$A_{k} \quad$ is the input matrix:

$$
A_{k}=\left(\begin{array}{cc}
\cos \left(\theta_{k / k}+\frac{\omega_{k}}{2}\right) & 0 \\
\sin \left(\theta_{k / k}+\frac{\omega_{k}}{2}\right) & 0 \\
0 & 0 \\
0 & 1 \\
0 & 0 \\
0 & 0
\end{array}\right)
$$

$u_{k} \quad$ is the input vector, it consists of the elementary displacement and elementary rotation of the vehicle obtained from the encoders measurements: $u=$ $[\Delta, \omega]$

$$
\begin{aligned}
\Delta_{k} & =\frac{r_{r} \Delta q r+r_{l} \Delta q l}{2} \\
\omega_{k} & =\frac{r_{r} \Delta q r-r_{l} \Delta q l}{e}
\end{aligned}
$$

$\Delta q r$ and $\Delta q l$ correspond to the elementary rotations of the right and left wheels respectively (expressed in radians), between the two sampling instants $k$ and $k+1$.

$e$ is the separation distance between the wheels. $r_{r}$ and $r_{l}$ represent respectively the radius of the right and the left wheels (we assume $r_{l}=r_{r}$ ).

$w_{k}$ is the process noise modeled as Gaussian white noise with zero mean and covariance matrix $Q_{k}$.

The model is non linear, therefore the EIF is applied. For this purpose, the Jacobian matrix $F_{k}=\left.\frac{\partial f}{\partial X}\right|_{X=X_{k / k}}$ and $B_{k}=$ $\left.\frac{\partial f}{\partial u}\right|_{u=u_{k}}$ are calculated: 


$$
\begin{aligned}
F_{k} & =\left(\begin{array}{cccccc}
1 & 0 & 0 & -\Delta_{k} \sin \left(\theta_{k / k}+\frac{\omega_{k}}{2}\right) & 0 & 0 \\
0 & 1 & 0 & \Delta_{k} \cos \left(\theta_{k / k}+\frac{\omega_{k}}{2}\right) & 0 & 0 \\
0 & 0 & 1 & 0 & 0 & 0 \\
0 & 0 & 0 & 1 & 0 & 0 \\
0 & 0 & 0 & 0 & 1 & T_{e} \\
0 & 0 & 0 & 0 & 0 & 1
\end{array}\right) \\
B_{k} & =\left(\begin{array}{cc}
\cos \left(\theta_{k / k}+\frac{\omega_{k}}{2}\right) & -\frac{1}{2} \Delta_{k} \sin \left(\theta_{k / k}+\frac{\omega_{k}}{2}\right) \\
\sin \left(\theta_{k / k}+\frac{\omega_{k}}{2}\right) & \frac{1}{2} \Delta_{k} \cos \left(\theta_{k / k}+\frac{\omega_{k}}{2}\right) \\
0 & 0 \\
0 & 1 \\
0 & 0
\end{array}\right.
\end{aligned}
$$

The corresponding covariance matrix can be written as:

$$
P_{k+1 / k}=F_{k} P_{k / k} F_{k}^{T}+B_{k}\left(Q_{u}\right)_{k} B_{k}^{T}+Q_{k}
$$

Where:

$Q_{u} \quad$ is the covariance matrix associated to the input vector $u_{k}$.

Consequently, the information matrix and information vector are obtained from the state vector and covariance matrix as follows:

$$
\begin{gathered}
Y_{k+1 / k}=P_{k+1 / k}^{-1} \\
y_{k+1 / k}=Y_{k+1 / k} X_{k+1 / k}
\end{gathered}
$$

\section{B. Update step}

The fusion of the pseudorange with the encoders measurements is done through a tightly coupled architecture using the EIF. Note that we have used the GPS (Global Positioning System) constellation [28] [29].

The pseudorange measurements can be modeled according to equation [13] [30] [2]:

$$
\rho_{i}=r_{i}+c \delta t_{r}+\epsilon_{i}
$$

where:

$\rho_{i}$ is the measured pseudorange

$r_{i}$ is the geometric distance between the satellite $i$ and the receiver

$\delta t_{r}$ is the receiver clock offset

$\epsilon_{i}$ is the noise errors after atmospheric and clock correction

$$
r_{i}=\sqrt{\left[x-x_{i}\right]^{2}+\left[y-y_{i}\right]^{2}+\left[z-z_{i}\right]^{2}}
$$

where $[x, y, z]$ is the receiver position and $\left[x_{i}, y_{i}, z_{i}\right]$ is the position of the satellite $i$ in the ECEF frame.

The observation equation (equation (13) can be expressed as:

$$
Z_{k}=h_{k}\left(X_{k}\right)+v_{k}
$$

where $v_{k}$ is the noise associated to the pseudorange measurements modeled as white Gaussian noise with zero mean and covariance matrix $R$.
The observation model is non linear, its linearization around the predicted estimation yields to:

$$
Z_{k}=h_{k}\left(X_{k / k-1}\right)+H_{k} \Delta X_{k}+v_{k}
$$

where $H$ is the Jacobian matrix given by:

$$
H_{k}=\left(\begin{array}{cccccc}
\frac{x_{k / k-1}-x_{1}}{r_{1}} & \frac{y_{k / k-1}-y_{1}}{r_{1}} & \frac{z_{k / k-1}-z_{1}}{r_{1}} & 0 & 1 & 0 \\
\frac{x_{k / k-1}-x_{2}}{r_{2}} & \frac{y_{k / k-1}-y_{2}}{r_{2}} & \frac{z_{k / k-1}-z_{2}}{r_{2}} & 0 & 1 & 0 \\
\vdots & & & & & \\
\frac{x_{k / k-1}-x_{N}}{r_{N}} & \frac{y_{k / k-1}-y_{N}}{r_{N}} & \frac{z_{k / k-1}-z_{N}}{r_{N}} & 0 & 1 & 0
\end{array}\right)_{k}^{k}
$$

The information matrix and the information vector are updated as shown in equations (18) and (19) respectively:

$$
\begin{aligned}
& Y_{k / k}=Y_{k / k-1}+\sum_{i=1}^{N} I_{i}(k) \\
& y_{k / k}=y_{k / k-1}+\sum_{i=1}^{N} i_{i}(k)
\end{aligned}
$$

where:

$$
\begin{gathered}
I_{i}(k)=H_{i, k}^{T} R_{i}^{-1}(k) H_{i, k} \\
i_{i}(k)=H_{i, k}^{T} R_{i}^{-1}(k)\left[\left(Z_{i, k}-\widehat{Z}_{i, k}\right)+H_{i, k} X_{k / k-1}\right]
\end{gathered}
$$

are the information contributions of the satellite measurement $i$,

$$
\begin{aligned}
& \widehat{Z}_{i, k}= \\
& \sqrt{\left[x_{k / k-1}-x_{i}\right]^{2}+\left[y_{k / k-1}-y_{i}\right]^{2}+\left[z_{k / k-1}-z_{i}\right]^{2}} \\
& +c \delta t_{r, k}
\end{aligned}
$$

is the estimated pseudorange measurement $i$,

$N$ is the number of satellites in view,

$H_{i}$ is the line $i$ of the matrix $H$ (equation 17 ).

\section{FAult Detection AND EXClusion BASED ON Weighted Kullbak-Leibler Divergence}

\section{A. Fault detection}

In our previous work [20], a residual based on the KullbackLeibler divergence, namely $G K L D$, was proposed:

$$
\begin{aligned}
G K L D= & \frac{1}{2} \operatorname{trace}\left(Y_{k / k} Y_{k / k-1}^{-1}\right)+\frac{1}{2} \log \frac{\left|Y_{k / k-1}\right|}{\left|Y_{k / k}\right|}-\frac{1}{2} M \\
& +\frac{1}{2}\left(X_{k / k}-X_{k / k-1}\right)^{T} Y_{k / k}\left(X_{k / k}-X_{k / k-1}\right)
\end{aligned}
$$

$M$ is the dimension of the state vector.

This residual is obtained from the calculation of the Kullback-Leibler divergence between two Gaussian distributions and it evaluates the divergence between the predicted and the updated probability distributions obtained from the EIF. 
It consists of two parts:

1) The Mahalanobis distance $\left(X_{k / k}-X_{k / k-1}\right)^{T} Y_{k / k}\left(X_{k / k}-X_{k / k-1}\right)$ : allows a test on the means

2) The Burg matrix divergence $\operatorname{trace}\left(\begin{array}{ll}Y_{k / k} & Y_{k / k-1}^{-1}\end{array}\right)+$ $\log \frac{\left|Y_{k / k-1}\right|}{\left|Y_{k / k}\right|}-M$ : allows a test on the covariance matrix.

However, with this representation, we have supposed the same confidences on the Mahalanobis distance and the Burg matrix divergence by the use of the weight 0.5 . Depending on the update distribution, we can specify a weight $\alpha$ that gives a visualization about the confidence on the pose estimation or on the covariance matrix. In fact, the trace of the covariance matrix represents the sum of the variance of the state vector estimation. If the value of the trace is high, this reflects an important variance on the pose estimation. Therefore, the confidence on the means values of the position must be weakened and that on the Burg matrix divergence should be increased. On the other hand, if the trace is low, the confidence on the means must be increased relatively to the one associated to the Burg matrix divergence.

Notice that the sum of confidences should be equal to one:

- $\alpha$ represents the confidence on the Burg matrix divergence

- $1-\alpha$ represents the confidence on the Mahalanobis distance. (24):

Therefore, we propose to express the weight as in equation

$$
\alpha=\frac{\operatorname{trace}\left(P_{k / k}\right)}{C}
$$

where $C$ is the maximal value of the $\operatorname{trace}\left(P_{k / k}\right)$ fixed according to the maximal variance on the state vector. It depends on the used GNSS receiver and the maximum allowed error.

The Weighted Kullback-Leibler Divergence $(W K L D)$ is given in equation (25):

$W K L D=\alpha\left[\operatorname{trace}\left(\begin{array}{ll}Y_{k / k} & Y_{k / k-1}^{-1}\end{array}\right)+\log \frac{\left|Y_{k / k-1}\right|}{\left|Y_{k / k}\right|}-M\right]$ $+(1-\alpha)\left[\left(X_{k / k}-X_{k / k-1}\right)^{T} Y_{k / k}\left(X_{k / k}-X_{k / k-1}\right)\right]$

The data distribution of the $W K L D$ can be obtained from the statistical study realized on the $G K L D[20]$ by replacing the weight $\frac{1}{2}$ by its corresponding value $\alpha$ or $1-\alpha$. Therefore, the distribution of the $W K L D$ can be expressed in function of the $F$-distribution and the Chi-square distributions:

$$
\begin{aligned}
& W K L D \sim(1-\alpha)\left[\frac{M(n-1)}{(n-M) n} F_{M, n-M}\right] \\
& +\alpha\left[\frac{1}{n-1} \frac{1}{1-\frac{1}{6(n-1)-1}\left(2 M+1-\frac{2}{M+1}\right)} \chi_{\frac{1}{2}(M(M+1))}^{2}\right]
\end{aligned}
$$

\section{B. Fault exclusion}

When a fault is detected using the $W K L D$, the faulty measurements should be excluded from the fusion procedure. For this reason, $N$ informational filters $\left(E I F_{j}\right)$ are designed. Each filter permits to calculate the information contribution of one observation (pseudorange). Therefore, from each filter $E I F_{j}$, a residual denoted $W K L_{j}$ can be calculated (figure 1 . It represents the divergence between the predicted distribution and the updated one obtained after the fusion of the pseudorange observation of satellite $j$. Notice that the calculation of the position estimation using one satellite is possible because we are in a tightly coupled architecture.

The residual $W K L_{j}$ is obtained as follows:

$W K L_{j, k}=$

$\alpha\left[\operatorname{trace}\left(Y_{j, k / k} Y_{k / k-1}^{-1}\right)+\log \frac{\left|Y_{k / k-1}\right|}{\left|Y_{j, k / k}\right|}-M\right]$
$+(1-\alpha)\left[\left(X_{j, k / k}-X_{k / k-1}\right)^{T} Y_{j, k / k}\left(X_{j, k / k}-X_{k / k-1}\right)\right]$

with:

$$
\begin{aligned}
Y_{j, k / k} & =Y_{k / k-1}+I_{j}(k) \\
y_{j, k / k} & =y_{k / k-1}+i_{j}(k) \\
X_{j, k / k} & =\left(Y_{j, k / k}\right)^{-1} y_{j, k / k}
\end{aligned}
$$

If the satellite measurement $n$ is faulty, The different $W K L_{j}$ will be lower than a threshold value expect $W K L_{n}$ (the only residual that contains the erroneous measurement). Therefore, while the $W K L D$ exceeds the threshold value, the measurement that leads to the maximum $W K L_{i}$ will be excluded from the fusion procedure. This iterative procedure will be repeated until no fault is detected using the $W K L D$. In order to avoid the exclusion of a healthy measurement, we introduce iteratively the excluded observations and we recalculate the $W K L D$. The set that passes the GT on the $W K L D$ will be used for the state estimation.

This approach that uses the EIF and the Kullback-Leibler divergence for the detection and exclusion of erroneous measurements, presents many advantages:

- The simplified model of the update step of EIF permits to add or to remove easily a pseudorange measurement. The fault tolerance aspect does not induce a high computational cost.

- The adopted model for the system allowed us to work in the state space. A fault which does not have a direct impact on the position of the vehicle will not be isolated. Indeed, in some cases, using measurements with errors could be a better alternative than their isolation in order to ensure an acceptable geometry of observations.

- In comparison with the MSS method which operates also in the state space, the latter needs a bank of filters for the faults detection and another bank for the exclusion of one fault. The exclusion of multiple faults will generate a high computational cost. 


\section{THRESHOLD OPTIMIZATION}

After the construction of the residual, its comparison with a threshold value is necessary in order to detect the presence of erroneous measurements. Classical FDE approaches for GNSS observations are based on fixing a false alarm probability [31] [32]. In aeronautic domain, the choice of false alarm and miss detection probabilities is done with agreement with the integrity risk standardized by ICAO. However, in urban applications, this choice is done heuristically. In this work, we propose more elaborate method for the threshold optimization based on the Kullback-Leibler Criterion $K L C$ (proposed in our previous work given in [20]). It is based on maximizing the information gain given by decision on the true hypothesis.

\section{A. Kullback-Leibler Criterion for threshold optimization}

We begin by some definitions:

$H_{0}$ and $H_{1}$ are the hypotheses of absence and presence of defaults respectively

$u_{0}$ and $u_{1}$ are the decisions of choosing $H_{0}$ and $H_{1}$ respectively

$P_{D}=p\left(u_{1} / H_{1}\right)$ is the detection probability: the probability of choosing $H_{1}$ when $H_{1}$ is true

$P_{F}=p\left(u_{1} / H_{0}\right)$ is the false alarm probability: the probability of choosing $H_{1}$ when $H_{0}$ is true

$P_{m d}=p\left(u_{0} / H_{1}\right)$ is the missed detection probability: the probability of choosing $H_{0}$ when $H_{1}$ is true

The priori probabilities are defined as:

$$
\begin{gathered}
p\left(H_{0}\right)=P_{0} \\
p\left(H_{1}\right)=1-P_{0}
\end{gathered}
$$

The Kullback-Leibler Summation is given by:

$$
K L S=K L S_{0}+K L S_{1}
$$

where

$$
\begin{aligned}
K L S_{j} & =K L\left(p\left(H / u_{j}\right) \| p(H)\right) \\
& =\sum_{i=\{0,1\}} p\left(H_{i} / u_{j}\right) \log \frac{p\left(H_{i} / u_{j}\right)}{p\left(H_{i}\right)}
\end{aligned}
$$

$K L S_{j}$ represents the information gain associated to the decision $u_{j}, j=0,1$.

$$
\begin{aligned}
K L S_{j} & =\frac{\alpha_{j}}{\alpha_{j}+\beta_{j}} \log \frac{\alpha_{j}}{P_{0}\left(\alpha_{j}+\beta_{j}\right)} \\
& +\frac{\beta_{j}}{\alpha_{j}+\beta_{j}} \log \frac{\beta_{j}}{\left(1-P_{0}\right)\left(\alpha_{j}+\beta_{j}\right)}
\end{aligned}
$$

Therefore $K L S$ can be written as:

$$
\begin{aligned}
K L S & =\frac{\alpha_{0}}{\alpha_{0}+\beta_{0}} \log \frac{\alpha_{0}}{P_{0}\left(\alpha_{0}+\beta_{0}\right)} \\
& +\frac{\beta_{0}}{\alpha_{0}+\beta_{0}} \log \frac{\beta_{0}}{\left(1-P_{0}\right)\left(\alpha_{0}+\beta_{0}\right)} \\
& +\frac{\alpha_{1}}{\alpha_{1}+\beta_{1}} \log \frac{\alpha_{1}}{P_{0}\left(\alpha_{1}+\beta_{1}\right)} \\
& +\frac{\beta_{1}}{\alpha_{1}+\beta_{1}} \log \frac{\beta_{1}}{\left(1-P_{0}\right)\left(\alpha_{1}+\beta_{1}\right)}
\end{aligned}
$$

where:

$$
\begin{array}{cc}
\alpha_{0}=P_{0}\left(1-P_{F}\right) & \beta_{0}=\left(1-P_{0}\right)\left(1-P_{D}\right) \\
\alpha_{1}=P_{0} P_{F} & \beta_{1}=\left(1-P_{0}\right) P_{D}
\end{array}
$$

In [20], we have demonstrated that the minimum of $K L S$ is obtained when $p(H / u)=p(H)$ indicating that the decision does not provide any information about the true hypothesis. Similarly, $K L S$ is minimum when $P_{F}=P_{D}$. However, maximizing the information gain given by the decision on the true hypothesis will lead to the maximum value of $K L S$.

Consequently, the Kullback-Leibler Criterion is defined as:

$$
K L C=\max (K L S)
$$

The likelihood ratio test obtained from this optimization problem (the maximization of $K L S$ ) is given by [20]:

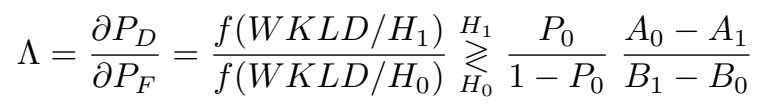

where

$$
\begin{aligned}
& A_{i}=\frac{\beta_{i}}{\left(\alpha_{i}+\beta_{i}\right)^{2}} \log \left(\frac{1-P_{0}}{P_{0}} \frac{\alpha_{i}}{\beta_{i}}\right) \\
& B_{i}=\frac{\alpha_{i}}{\left(\alpha_{i}+\beta_{i}\right)^{2}} \log \left(\frac{P_{0}}{1-P_{0}} \frac{\beta_{i}}{\alpha_{i}}\right) \\
& i=0,1
\end{aligned}
$$

Therefore, the optimal threshold is obtained from the values of $P_{F}$ and $P_{D}$ that maximize $K L S$. For this purpose, the probabilities density functions ( $p d f)$ of the $W K L D$ in the faulty $\left(f\left(W K L D / H_{1}\right)\right)$ and non faulty cases $\left(f\left(W K L D / H_{0}\right)\right)$ should be known.

The decision criterion can be expressed directly in function of the $W K L D$ as:

$W K L D \underset{H_{0}}{\stackrel{H_{1}}{\gtrless}} \lambda=$ threshold

= function of $\left(A_{i}, B_{i}, P_{0}\right.$, parameters of the $p d f$ of the $\left.W K L D\right)$

Notice, that $K L S$ is function of the priori probability $P_{0}$. In order to avoid a heuristic choice of this probability, $P_{0}$ will be calculated based on the Maximum Likelihood Estimation (MLE) and using the historical system behavior:

$$
\widehat{P}_{0}=1-\frac{\sum_{i=1}^{n} h^{i}}{n}
$$

where $h=\left(h^{1}, h^{2}, \ldots, h^{n}\right)$ is a random samples of chosen hypotheses and:

$$
h^{i}=0 \text { if } H_{0} \text { is chosen }
$$$$
h^{i}=1 \text { if } H_{1} \text { is chosen }
$$

In this work, the sample of hypotheses at instant $k$ is obtained from the decisions taken from the sampling instant $k_{1}$ to $k-1$, where $\left[k_{1}, k-1\right]$ is a sliding interval with width equal to 50 sampling instants and

$$
\begin{cases}k_{1}=1 & \text { if } k \leq 50 \\ k_{1}=k-50 & \text { if } k>50\end{cases}
$$




\section{B. Interpretation of $K L S$}

The $K L S$ can be viewed as the summation of $K L S_{0}$ and $K L S_{1}$ (equations 35 and 36). The maximization of $K L S_{0}$ is equivalent to maximizing the information gain given by the decision $u_{0}$ (no fault). This gain is maximum if we are sure that the true hypothesis is $H_{0}$. Therefore, this optimization problem leads to a threshold denoted $t h_{K L C_{0}}$ : it's a liberal threshold (figure 2 .

The threshold that maximizes $K L S_{0}$ is obtained by setting the derivative of $K L S_{0}$ to 0 : $\frac{\partial K L S_{0}}{\partial v}=0$ where $v$ denotes the threshold. The likelihood ratio test obtained from this optimization problem is:

$$
\Lambda_{0}=\frac{f\left(W K L D / H_{1}\right)}{f\left(W K L D / H_{0}\right)} \underset{H_{0}}{\gtrless} \frac{-P_{0}}{1-P_{0}} \frac{A_{0}}{B_{0}}
$$

where:

$$
\frac{A_{0}}{B_{0}}=-\frac{\beta_{0}}{\alpha_{0}}
$$

Consequently:

$$
\Lambda_{0}=\frac{f\left(W K L D / H_{1}\right)}{f\left(W K L D / H_{0}\right)} \underset{H_{0}}{\stackrel{H_{1}}{\gtrless}} \frac{1-P_{D}}{1-P_{F}}
$$

Demonstration: After a simple calculation and using equation (46) in reference [20] we can obtain:

$$
\frac{\partial K L S_{0}}{\partial v}=\frac{\partial \alpha_{0}}{\partial v} A_{0}+\frac{\partial \beta_{0}}{\partial v} B_{0}=0
$$

Therefore:

$$
P_{0} \frac{\partial P_{F}}{\partial v} A_{0}=-\left(1-P_{0}\right) \frac{\partial P_{D}}{\partial v} B_{0}
$$

Which leads:

$$
\frac{\partial P_{D}}{\partial P_{F}}=\frac{-P_{0}}{1-P_{0}} \frac{A_{0}}{B_{0}}
$$

Likewise, the maximization of $K L S_{1}$ is equivalent to maximizing the information gain given by the decision $u_{1}$ (fault). This gain is maximum if we are sure that the true hypothesis is $H_{1}$. Therefore, this optimization problem leads to a threshold denoted $t h_{K L C_{1}}$ : it is a conservative one (figure 2). The likelihood ratio test obtained from the maximization of $K L S_{1}$ is:

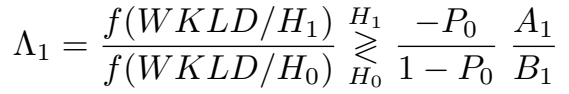

with:

$$
\frac{A_{1}}{B_{1}}=-\frac{\beta_{1}}{\alpha_{1}}
$$

Consequently:

$$
\Lambda_{1}=\frac{f\left(W K L D / H_{1}\right)}{f\left(W K L D / H_{0}\right)} \underset{H_{0}}{\stackrel{H_{1}}{\gtrless}} \frac{P_{D}}{P_{F}}
$$

It can be noticed that the thresholds $t h_{K L C_{0}}$ and $t h_{K L C_{1}}$ are independent from the priori probability $P_{0}$.

Given that we are interested by the case where $P_{D}>P_{F}$, we have:

$$
\frac{P_{D}}{P_{F}}>1
$$

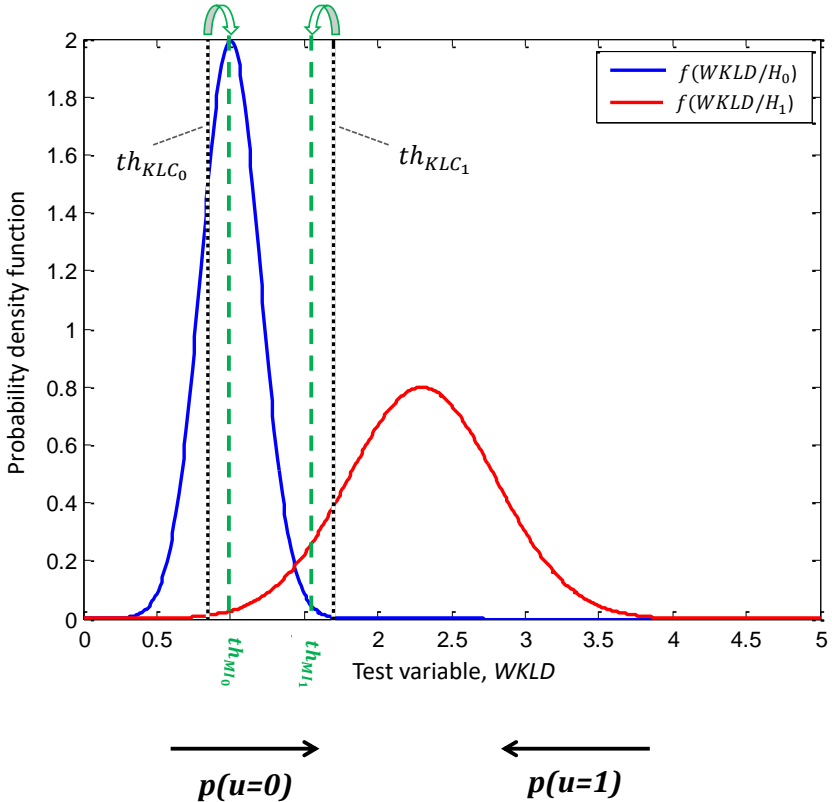

Fig. 2: The interval of thresholds using the $K L C$ and the $M I$.

and

$$
\frac{1-P_{D}}{1-P_{F}}<1
$$

which verifies our interpretation that $t h_{K L C_{0}}<t h_{K L C_{1}}$

According to the application, the user can choose a liberal threshold, a conservative threshold or a threshold varying with a system parameter. The threshold that maximizes $K L S$ can varies from $t h_{K L C_{0}}$ to $t h_{K L C_{1}}$ and is influenced by the value of the priori probability $P_{0}$.

For a large value of $P_{0}$, the threshold will approach $t h_{K L C_{1}}$ since $\max \left(K L S_{1}\right) \gg \max \left(K L S_{0}\right)$. Because, in this case, the decision $u_{1}$ provides the maximum information gain, therefore

$$
\max \left(K L S_{0}+K L S_{1}\right) \longrightarrow \max \left(K L S_{1}\right) .
$$

\section{Comparison with the Mutual Information Criterion}

The Mutual Information $(M I)$ criterion for threshold optimization was proposed in [33] [34]. The goal was to minimize the uncertainty between the decision and the hypothesis which is equivalent to maximize the $M I$.

The likelihood ratio test obtained from this optimization problem is given by:

$$
\Lambda=\frac{f\left(W K L D / H_{1}\right)}{f\left(W K L D / H_{0}\right)} \underset{H_{0}}{\gtrless} \frac{P_{0}}{1-P_{0}} \frac{C_{10}-C_{00}}{C_{01}-C_{11}}
$$

where:

$$
C_{i j}=\log \left(\frac{p\left(u_{i}, H_{j}\right)}{p\left(u_{i}\right) p\left(H_{j}\right)}\right)=\log \left(\frac{p\left(u_{i} / H_{j}\right)}{p\left(u_{i}\right)}\right)
$$

In this section, we propose to decompose the $M I$ criterion into two criteria and to compare the obtained interval to that of $K L S$. 
The $M I(I(H, u))$ can be viewed as the expected value of the $K L S$, indeed:

$$
\begin{aligned}
I(H, u) & =\sum_{H} \sum_{u} p(H / u) p(u) \log \frac{p(H / u)}{p(H)} \\
& =\sum_{u} p(u) \sum_{H} p(H / u) \log \frac{p(H / u)}{p(H)} \\
& =\sum_{u} p(u) K L(p(H / u), p(H)) \\
& =E_{u}[K L(p(H / u), p(H))]
\end{aligned}
$$

Consequently, $I(H, u)$ can be expressed in function of $K L S_{0}$ and $K L S_{1}$ as follows:

$$
I(H, u)=p(u=0) K L S_{0}+p(u=1) K L S_{1}
$$

Equation (58) can be considered as the summation of two terms:

$$
\begin{aligned}
M I_{0} & =p(u=0) K L S_{0} \\
& =\alpha_{0} \log \frac{\alpha_{0}}{P_{0}\left(\alpha_{0}+\beta_{0}\right)}+\beta_{0} \log \frac{\beta_{0}}{\left(1-P_{0}\right)\left(\alpha_{0}+\beta_{0}\right)}
\end{aligned}
$$

and

$$
\begin{aligned}
M I_{1} & =p(u=1) K L S_{1} \\
& =\alpha_{1} \log \frac{\alpha_{1}}{P_{0}\left(\alpha_{1}+\beta_{1}\right)}+\beta_{1} \log \frac{\beta_{1}}{\left(1-P_{0}\right)\left(\alpha_{1}+\beta_{1}\right)}
\end{aligned}
$$

Therefore, the corresponding likelihood ratio test obtained from the maximization of $M I_{0}$ is:

$$
\begin{aligned}
\Lambda_{0}=\frac{f\left(W K L D / H_{1}\right)}{f\left(W K L D / H_{0}\right)} \underset{H_{0}}{\stackrel{H_{1}}{\gtrless}}-\frac{P_{0}}{1-P_{0}} \frac{C_{00}}{C_{01}} \\
=-\frac{P_{0}}{1-P_{0}} \frac{\log \frac{\alpha_{0}}{P_{0}\left(\alpha_{0}+\beta_{0}\right)}}{\log \frac{\beta_{0}}{\left(1-P_{0}\right)\left(\alpha_{0}+\beta_{0}\right)}}
\end{aligned}
$$

Likewise, the maximization of $M I_{1}$ leads to the likelihood ratio test $\Lambda_{1}$ :

$$
\begin{aligned}
\Lambda_{1}=\frac{f\left(W K L D / H_{1}\right)}{f\left(W K L D / H_{0}\right)} \underset{H_{0}}{\stackrel{H_{1}}{\gtrless}}-\frac{P_{0}}{1-P_{0}} \frac{C_{10}}{C_{11}} \\
=-\frac{P_{0}}{1-P_{0}} \frac{\log \frac{\alpha_{1}}{P_{0}\left(\alpha_{1}+\beta_{1}\right)}}{\log \frac{\beta_{1}}{\left(1-P_{0}\right)\left(\alpha_{1}+\beta_{1}\right)}}
\end{aligned}
$$

Consequently, contrary to $K L S_{0}$ and $K L S_{1}$, the maximization of $M I_{0}\left(M I_{1}\right)$ leads to a threshold $t h_{M I_{0}}\left(t h_{M I_{1}}\right)$ depending on $P_{0}$. The interval $\left[t h_{M I_{0}}, t h_{M I_{1}}\right]$ varies with the value of $P_{0}$ which is not the case for the interval $\left[t h_{K L C_{0}}, t h_{K L C_{1}}\right]$.

On the other hand, the aim is to compare these two intervals. For this reason, the study of the variation of $p(u=0)$ and $p(u=1)$ is necessary:

$$
\begin{gathered}
p(u=0)=P_{0}\left(1-P_{F}\right)+\left(1-P_{0}\right)\left(1-P_{D}\right) \\
p(u=1)=P_{0} P_{F}+\left(1-P_{0}\right) P_{D}
\end{gathered}
$$

For a given value of $P_{0}, p(u=0)$ increases when the values of $P_{F}$ and $P_{D}$ decrease, so when the threshold value increases.
Likewise, $p(u=1)$ increases if $P_{F}$ and $P_{D}$ increase, so if the threshold value decreases. The maximum of $K L S_{0}$ is obtained at the value of $t h_{K L C_{0}}$. From figure 3 , we can remark that the maximum of $M I_{0}$ (i.e. $\left.p(u=0) K L S_{0}\right)$ can only move to the right of the one of $K L S_{0}$. Similarly, from figure 4, the maximum of $M I_{1}$ (i.e. $\left.p(u=1) K L S_{1}\right)$ can only move to the left of the one of $K L S_{1}$.
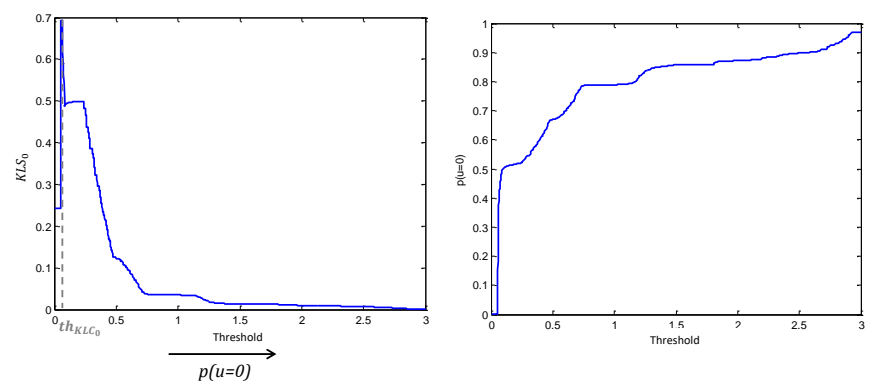

Fig. 3: Representation of $K L S_{0}$ and $p(u=0)$ in function of the threshold value.
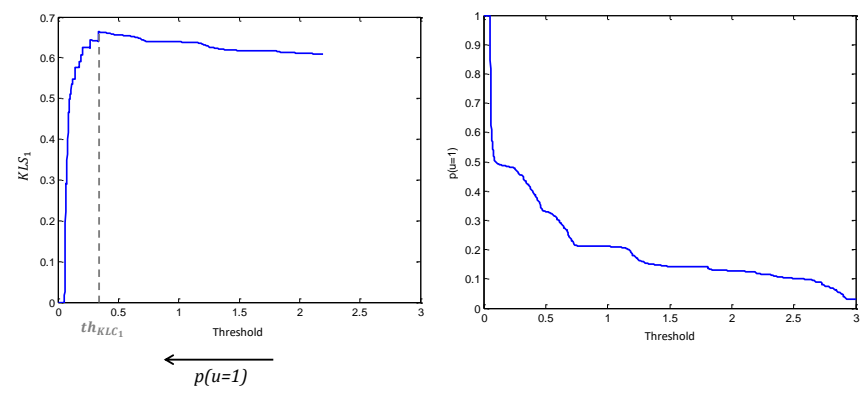

Fig. 4: Representation of $K L S_{1}$ and $p(u=1)$ in function of the threshold value.

The interval of thresholds obtained from $K L S$ and $M I$ are shown in figure 2. $\left[t h_{K L C_{0}}, t h_{K L C_{1}}\right]$ is a fixed interval and more conservative than $\left[t h_{M I_{0}}, t h_{M I_{1}}\right]$. The threshold obtained from the maximization of $K L S$ lies always inside this interval and varies from $t h_{K L C_{0}}$ to $t h_{K L C_{1}}$.

\section{EXPERIMENTATION AND RESULTS VALIDATION}

In order to test the performance of the proposed approach, experimentation with real data from a GPS constellation and Cycab vehicle are achieved (figure 5). Figure 6 shows the trajectory of the vehicle in the case of a tightly coupled GPS/odometer compared to the case when using only GPS measurements. The amelioration is clear especially in the region surrounded by trees.

The $W K L D$ that quantifies the divergence between the predicted and the updated distributions is shown in figure 7 It presents many jumps indicating the presence of erroneous GPS measurements: In presence of faults and after the update step, the GPS measurements lead to a position estimation that diverges from the one obtained using the odometer model (prediction step).

In order to detect the presence of erroneous measurements, the $W K L D$ should be compared to a threshold value. Consequently, the probability distributions of the $W K L D$ in the 


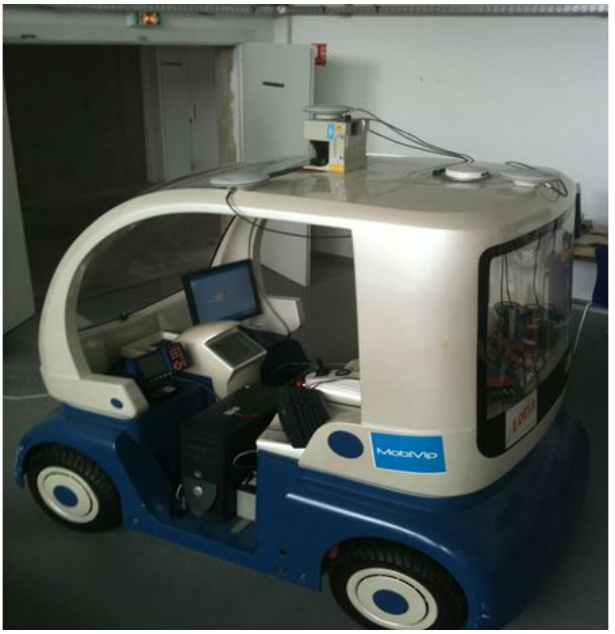

Fig. 5: The Cycab vehicle used for experimentation.

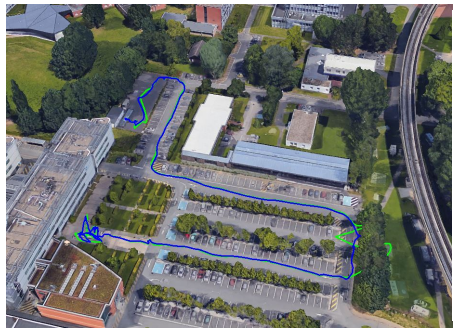

(a)

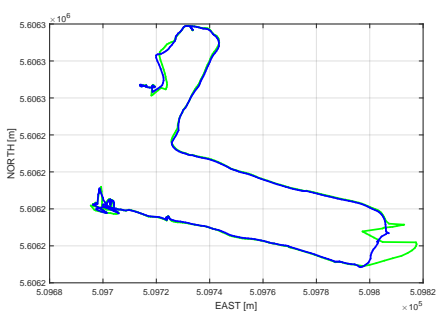

(b)
Fig. 6: Trajectory of the vehicle in the case of using GPS data (in green) and when using a tightly coupled architecture GPS/odometer (in blue).

faulty and non faulty cases should be determined previously. This can be achieved by integrating a learning step based on the reference data or using a simulator.

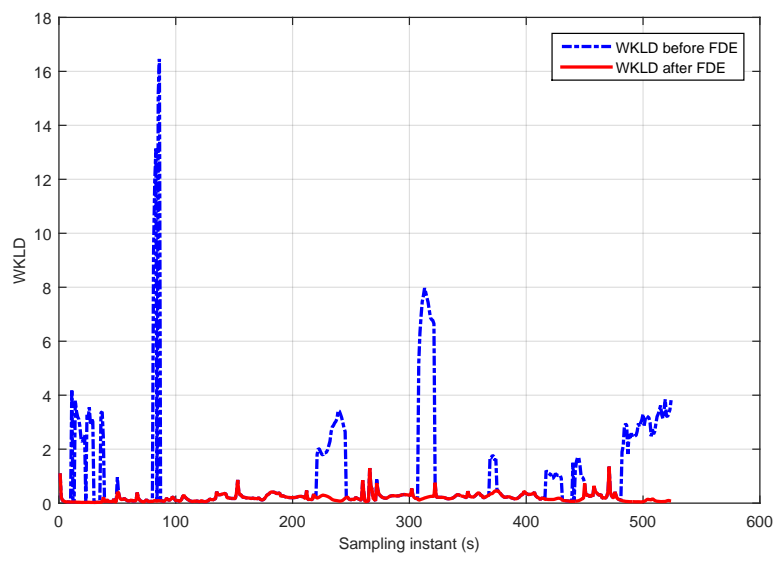

Fig. 7: The $W K L D$ before and after FDE step.

Figure $8 \mathrm{a}$ shows the empirical probability distributions of the $W K L D$ in the non faulty case after taking into account healthy pseudorange measurements. Figure $8 \mathrm{~b}$ presents the empirical probability distribution of the $W K L D$ in the faulty case. It is obtained after incorporating different amplitude values of the errors in the pseudorange measurements. These two distributions are exploited in order to determine the optimal threshold that maximizes the $K L S$. When $P_{0}$ is equal to 0.5 , the optimal threshold is obtained at $\lambda=0.901$ corresponding to a false alarm probability of 0.0041 and a detection probability of 0.8725 (figure 9 ).

The liberal and conservative thresholds obtained from the maximization of $K L S_{0}$ and $K L S_{1}$ respectively are shown in figure 10. $\left[t h_{K L C_{0}}, t h_{K L C_{1}}\right]$ forms an envelope in which the threshold varies (figure 11). The optimal threshold $\lambda$ lies inside this interval and depends on the value of $P_{0}$ obtained using the MLE and the historical system behavior under a sliding interval, as explained in section IV-A (figure 12.

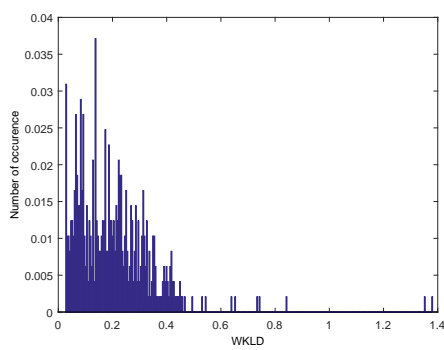

(a) Non-faulty case

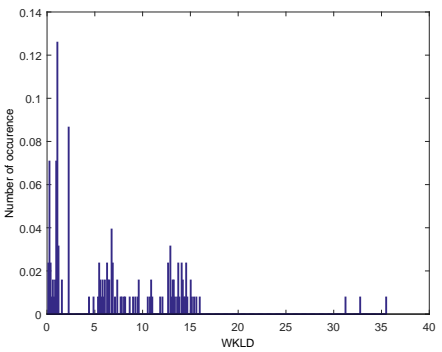

(b) Faulty case
Fig. 8: Data distributions of the $W K L D$ in the non faulty $8 \mathrm{a}$ and faulty $8 \mathrm{~b} b$ cases.

Figure 13 shows the $W K L_{i}$ used for the fault exclusion. Indeed, when the $W K L D$ exceeds the threshold value, the set of $W K L_{i}$ should be calculated in order to determine the origin of the faults. For example, the information contribution of satellite 9 (corresponding to the residual $W K L_{9}$ ) is incoherent with the others indicating that this satellite should be excluded from the fusion procedure at instants $k=15$ to $30, k=82$ to 87 and $k=483$ to $k=525 \mathrm{~s} \ldots$ In order to validate that the measurements of satellite 9 are erroneous, the elevation of the different satellites are shown in figure 14 . We can remark that the elevation of satellite 9 is about $6^{\circ}$ which increases the multipath probability and therefore the probability of errors.

In order to verify the aptitude of the method in detecting the erroneous measurements and specifically in detecting multiple faults at a time, we propose to simulate errors in the pseudor-

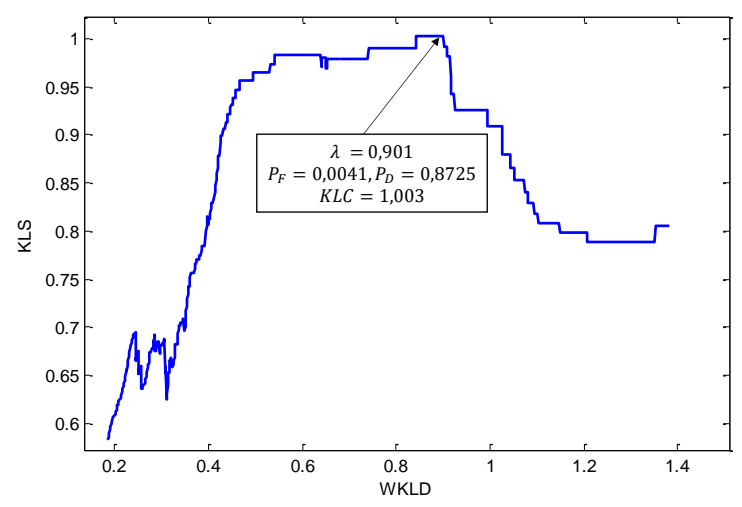

Fig. 9: The optimal threshold $\lambda$ when using $K L C$ for $P_{0}=$ 0.5 . 


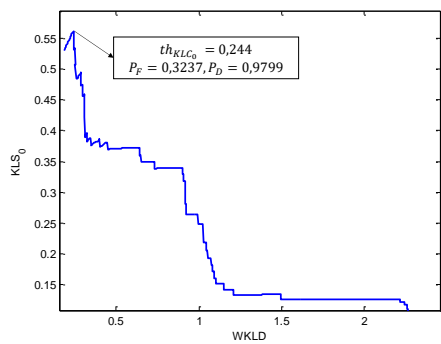

(a) $K L S_{0}$

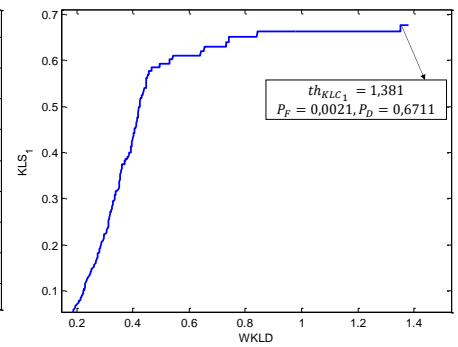

(b) $K L S_{1}$
Fig. 10: The liberal and conservative thresholds obtained from the maximization of $K L S_{0}$ and $K L S_{1}$.

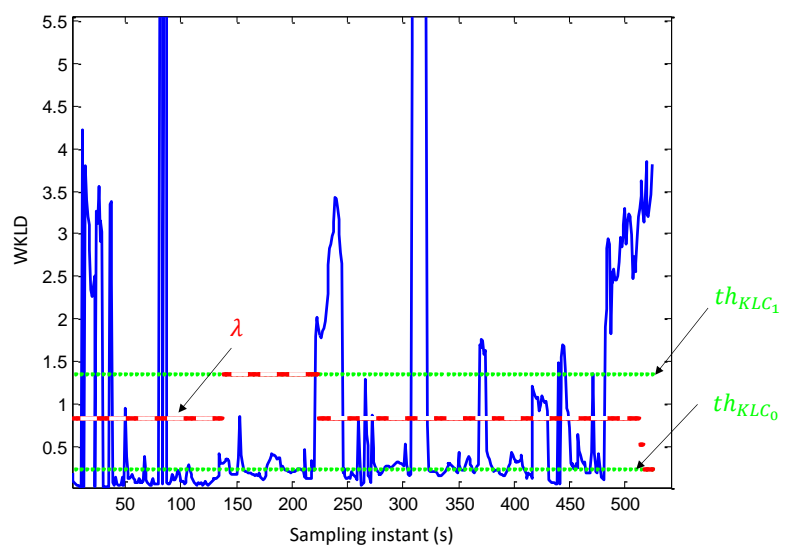

Fig. 11: The $W K L D$ with the corresponding threshold $\lambda$

ange measurements of satellite 12 from instant $k=222$ to 246 $\mathrm{s}$, and in the pseudorange measurements of satellites 4 and 12 from $k=309$ to $322 \mathrm{~s}$. Figure 13 shows the jumps that appear in $W K L_{12}$ from $k=222$ to 246 indicating that this satellite should be excluded during these sampling instants. Likewise, the jumps in $W K L_{12}$ and $W K L_{4}$ indicate that satellites 12 and 4 should be excluded from the fusion procedure from $k=309$ to 322 .

The estimated trajectories before and after the FDE step are

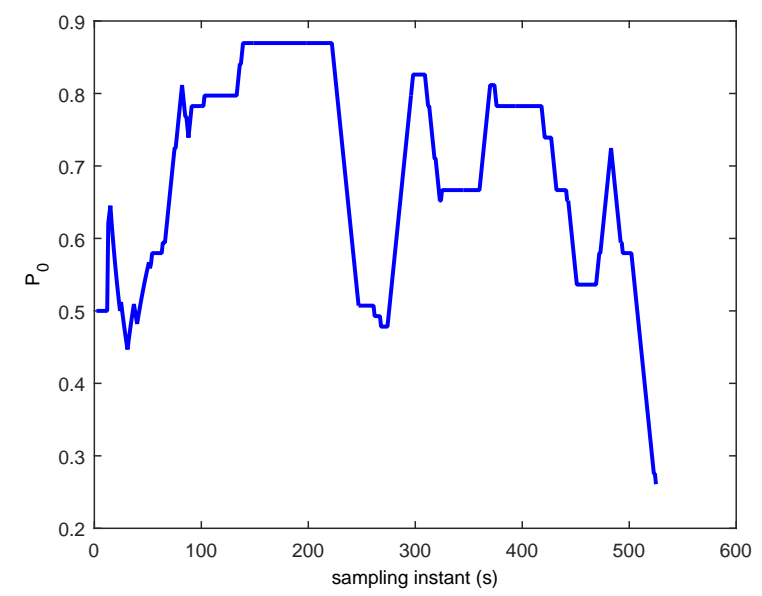

Fig. 12: The variation of the priori probability $P_{0}$ obtained using the MLE.

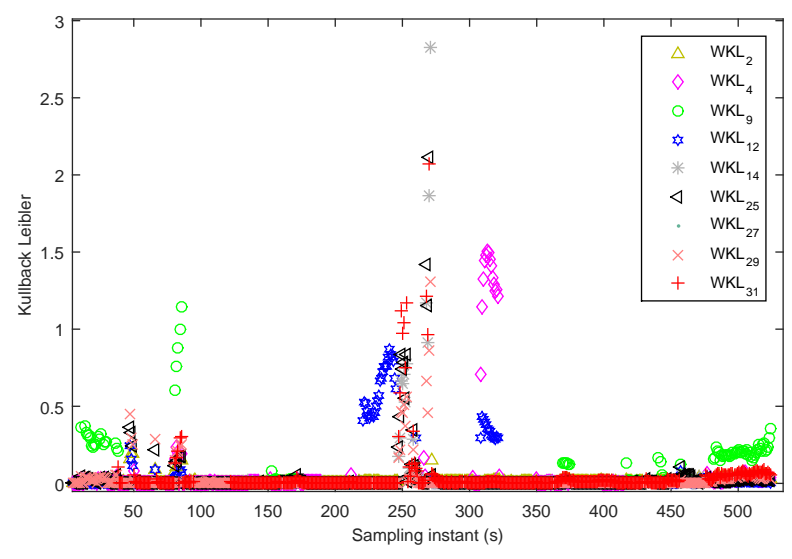

Fig. 13: The $W K L_{i}$ for the fault exclusion

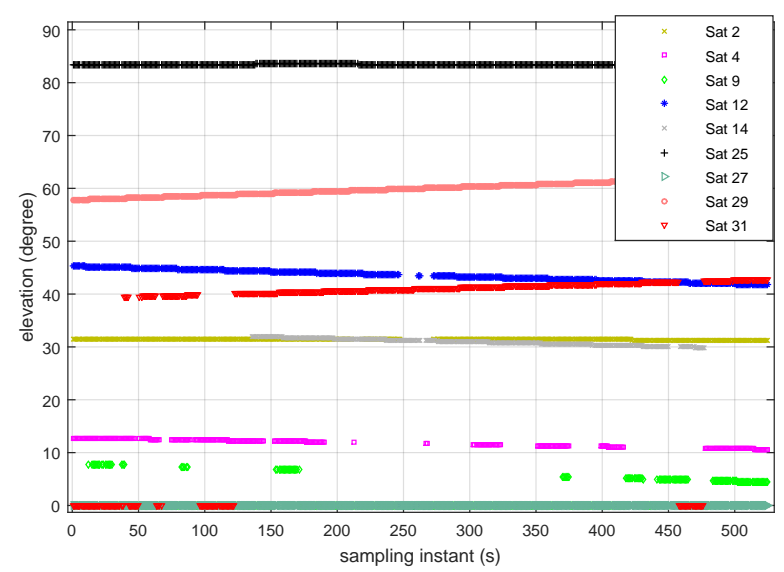

Fig. 14: The elevation of the satellites in view.

shown in figure 15 and 16 . It can be seen the improvement in the trajectory estimation after the FDE step. Indeed, it should be noted that the trajectory was degraded at the level of the estimation of the height (h) which presents some unjustifiable jumps before the exclusion of the erroneous satellite measurements.

Figure 17 shows the variations of the false alarm and

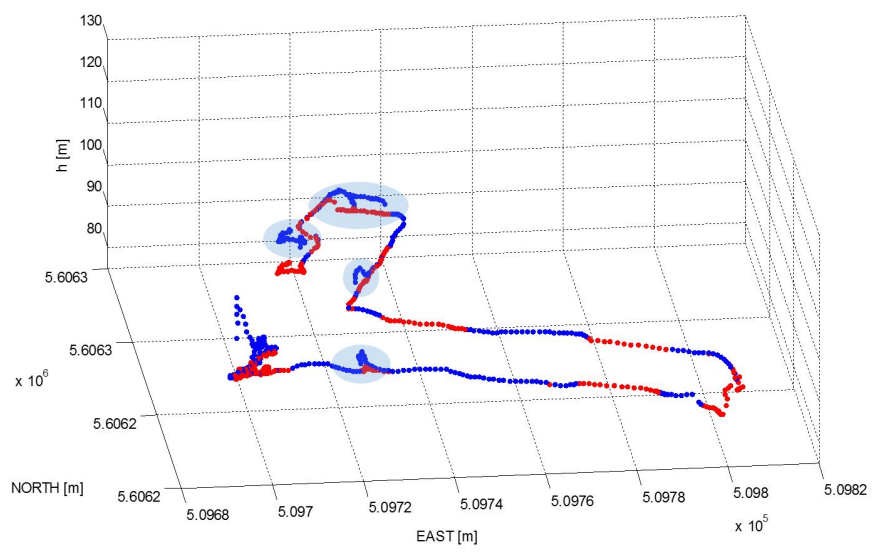

Fig. 15: 3D Trajectories before FDE (in blue) and after FDE (in red). 


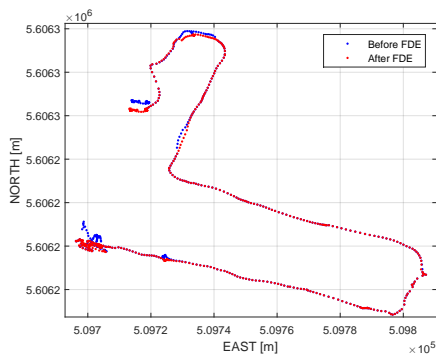

(a) Trajectory in 2D.

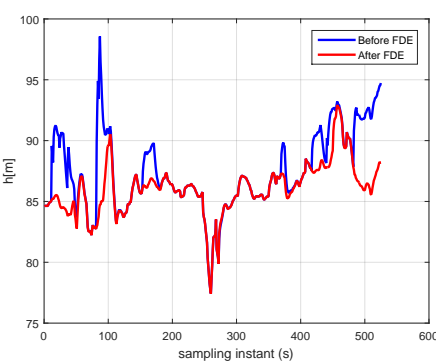

(b) The Height.
Fig. 16: The 2D trajectories and the height before FDE (in blue) and after FDE (in red).

detection probabilities in function of the priori probability $P_{0}$, obtained in the case of the $K L C$ and the $M I$ criterion. It can be remarked that for $P_{0}<0.5$ (hypothesis "presence of faults" is more probable), the $K L C$ leads to $P_{F}$ and $P_{D}$ higher than in the case of the $M I$ criterion ensuring better faults detection. However, for $P_{0}>0.5$ (hypothesis "absence of faults" is more probable), the $K L C$ reduces the false alarm probability leading to an automatic reduction of the detection probability compared to the $M I$ criterion. Therefore, the $K L C$ presents a better behavior in function of $P_{0}$.

\section{CONCLUSION}

In this paper, a tightly coupled GPS/odometer with faults diagnosis is proposed. The method is based on the use of the EIF for the state estimation and on the $W K L D$ for the faults detection. When a fault is detected using the $W K L D$, a set of residuals obtained from a bank of $E I F_{j}$ is generated in order to exclude the erroneous pseudorange measurements. Optimal thresholding method based on the Kullback-Leibler Criterion is proposed. The optimal threshold is the value that maximizes the information gain given by a decision and is influenced by the priori probability obtained using the MLE and the historical system behavior under a sliding interval.

Experimental results show that the method was able to localize accurately the vehicle while detecting and excluding the erroneous pseudorange measurements in a relevant manner. Likewise, the method ensures the exclusion of multiple faults at a given instant.

Comparison between the $K L C$ and the $M I$ criterion is given theoretically and then experimentally in order to show the performance of each criterion.

\section{ACKNOWLEDGMENT}

This work has been supported by the project ELSAT2020 by Cisit. This project is co-financed by the European Union with FEDER funds, by the French State and the Hauts de France Region.

\section{REFERENCES}

[1] V. Drevelle and P. Bonnifait, "Igps: Global positioning in urban canyons with road surface maps," IEEE Intelligent Transportation Systems Magazine, vol. 4, no. 3, pp. 6-18, 2012, ISSN: 1939-1390.
[2] V. Drevelle, "Étude de méthodes ensemblistes robustes pour une localisation multisensorielle intègre. application à la navigation des véhicules en milieu urbain.," $\mathrm{PhD}$ thesis, Université de Technologie de Compiègne, Dec. 1, 2011.

[3] A. Angrisano, "GNSS/INS integration methods," $\mathrm{PhD}$ thesis, universita' degli studi di napoli "Parthenope", 2010.

[4] Z. Tao, "Autonomous road vehicles localization using satellites, lane markings and vision," $\mathrm{PhD}$ thesis, Compiègne, 2016.

[5] J. Tang, Y. Chen, X. Niu, L. Wang, L. Chen, J. Liu, C. Shi, and J. Hyyppä, "LiDAR scan matching aided inertial navigation system in GNSS-denied environments," Sensors, vol. 15, no. 7, pp. 16 710-16 728, 2015.

[6] M. G. Petovello, "Real-time integration of a tacticalgrade IMU and GPS for high-accuracy positioning and navigation," $\mathrm{PhD}$ thesis, University of Calgary, 2003.

[7] U. I. Bhatti, "Improved integrity algorithms for integrated GPS/INS systems in the presence of slowly growing errors," $\mathrm{PhD}$ thesis, University of London, 2007.

[8] Y. W. Huang and K. W. Chiangb, "Improving the performance of MEMS IMU/GPS pos systems for land based MMS utilizing tightly coupled integration and odometer," in ISPRS proceedings, 2010.

[9] M. George and S. Sukkarieh, "Tightly coupled INS/GPS with bias estimation for UAV applications," in Proceedings of Australiasian Conference on Robotics and Automation (ACRA), 2005.

[10] I. Miller, B. Schimpf, M. Campbell, and J. Leyssens, "Tightly-coupled GPS / INS system design for autonomous urban navigation," in 2008 IEEE/ION Position, Location and Navigation Symposium, May 2008, pp. 1297-1310.

[11] B. W. Parkinson and P. Axelrad, "Autonomous GPS integrity monitoring using the pseudorange residual," Navigation, vol. 35, no. 2, pp. 255-274, Jun. 1, 1988, ISSN: 2161-4296.

[12] M. A. Sturza, "Navigation system integrity monitoring using redundant measurements," Navigation, vol. 35, no. 4, pp. 483-501, 1988.

[13] Y. C. Lee, "Analysis of range and position comparison methods as a means to provide GPS integrity in the user receiver," in Proceedings of the Annual Meeting of the Institute of Navigation, Citeseer, 1986, pp. 1-4.

[14] R. G. Brown and P. W. McBURNEY, "Self-contained GPS integrity check using maximum solution separation," Navigation, vol. 35, no. 1, pp. 41-53, Mar. 1, 1988, ISSN: 2161-4296.

[15] A. Martineau, "Etude de la performance du contrôle autonome d'intégrité pour les approches à guidage vertical," PhD thesis, Université de Toulouse, Nov. 14, 2008.

[16] H. Kuusniemi, A. Wieser, G. Lachapelle, and J. Takala, "User-level reliability monitoring in urban personal satellite-navigation," IEEE Transactions on Aerospace 


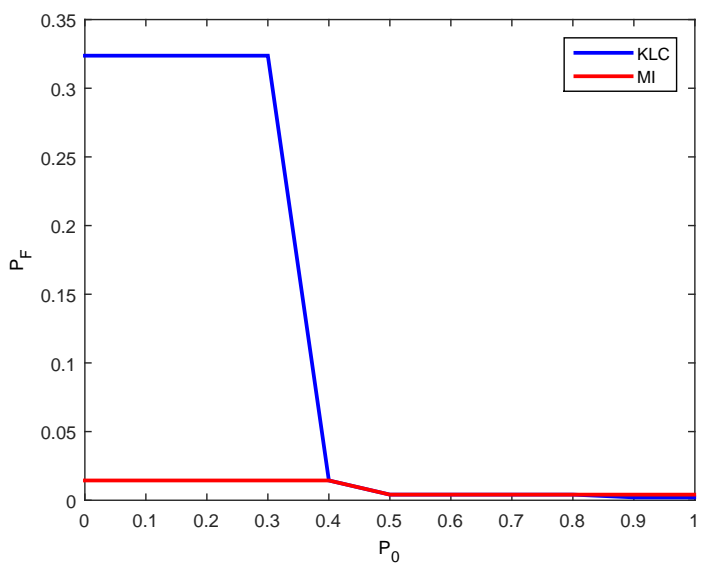

(a) $P_{F}$ in function of $P_{0}$.

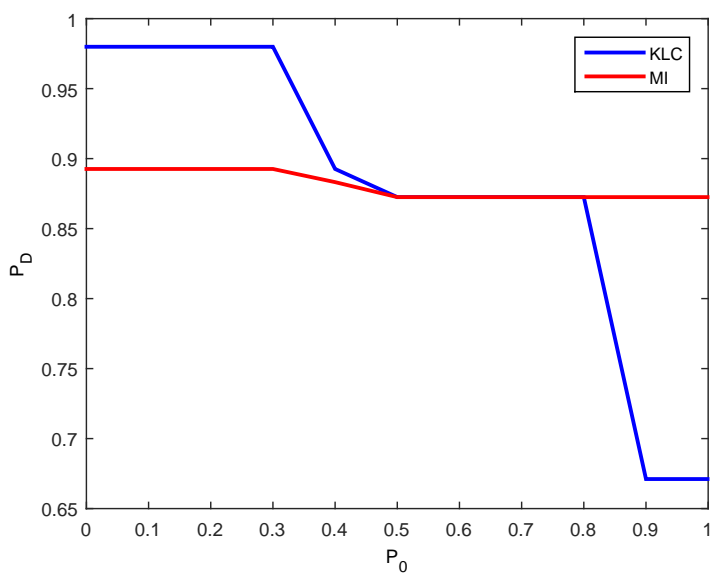

(b) $P_{D}$ in function of $P_{0}$.

Fig. 17: Variations of $P_{F}$ and $P_{D}$ in function of $P_{0}$ obtained using the $K L C$ and the $M I$ criterion.

and Electronic Systems, vol. 43, no. 4, pp. 1305-1318, 2007.

[17] A. Angrisano, C. Gioia, S. Gaglione, and G. Del Core, "GNSS reliability testing in signal-degraded scenario," International Journal of Navigation and Observation, vol. 2013, 2013.

[18] H. Kuusniemi and G. Lachapelle, "GNSS signal reliability testing in urban and indoor environments," in Proceedings of the NTM Conference, 2004.

[19] H. Durrant-Whyte, Introduction to decentralised data fusion. The University of Sydney, Sydney, Australia, 2004.

[20] J. Al Hage, M. E. El Najjar, and D. Pomorski, "Multisensor fusion approach with fault detection and exclusion based on the kullback-leibler divergence: Application on collaborative multi-robot system," Information Fusion, vol. 37, pp. 61-76, Sep. 2017, ISSN: 1566-2535.

[21] N. Assimakis, M. Adam, and A. Douladiris, "Information filter and kalman filter comparison: Selection of the faster filter," International Journal of Information Engineering, vol. 2, no. 1, pp. 1-5, 2012.

[22] M. Lexa, "Useful facts about the kullback-leibler discrimination distance," Rice University, Houston, 2004.

[23] M. N. Do, "Fast approximation of kullback-leibler distance for dependence trees and hidden markov models," IEEE signal processing letters, vol. 10, no. 4, pp. 115118, 2003.

[24] I. S. Dhillon and J. A. Tropp, "Matrix nearness problems with bregman divergences," SIAM Journal on Matrix Analysis and Applications, vol. 29, no. 4, pp. 11201146, 2007.

[25] International Civil Aviation Organization, International standards and recommended practices-annex 10 to the convention on international civil aviation- aeronautical telecommunications- volume $i$, radio navigation aids, Jul. 2006.

[26] I. Hozo, B. Djulbegovic, S. Luan, A. Tsalatsanis, and G. Gigerenzer, "Towards theory integration: Threshold model as a link between signal detection theory, fast- and-frugal trees and evidence accumulation theory," Journal of evaluation in clinical practice, 2016.

[27] F. W. Scholz, "Maximum likelihood estimation," in Encyclopedia of Statistical Sciences, John Wiley \& Sons, Inc., 2004, ISBN: 978-0-471-66719-3.

[28] Official U.S. Government information about the Global Positioning System (GPS) and related topics. (). GPS.gov: Space segment.

[29] E. Kaplan and C. Hegarty, Understanding GPS: Principles and applications. Artech house, 2005.

[30] F. Faurie, "Algorithmes de contrôle d'intégrité pour la navigation hybride GNSS et systèmes de navigation inertielle en présence de multiples mesures satellitaires défaillantes," PhD thesis, Bordeaux 1, 2011.

[31] A. Martineau, C. Macabiau, I. Nikiforov, and B. Roturier, "Performance of receiver autonomous integrity monitoring (RAIM) for vertically guided approaches," in ENC-GNSS 2008, Conférence Européenne de la Navigation, 2008.

[32] S. Andrés and C. Daniel, "Integrity monitoring applied to the reception of GNSS signals in urban environments," PhD thesis, Toulouse, INPT, 2012.

[33] P. K. Varshney, Distributed detection and data fusion. Springer Science \& Business Media, 2012.

[34] I. Y. Hoballah and P. K. Varshney, "An information theoretic approach to the distributed detection problem," IEEE Transactions on Information Theory, vol. 35, no. 5, pp. 988-994, 1989. 


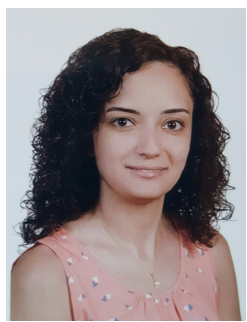

Joelle Al Hage received the degree in electrical and electronic engineering and the master degree in Reliability, Identification and Diagnosis from the Lebanese university in 2013. She received the Ph.D. degree in automatic and computer science from university of Lille in October 2016. In 2016/2017, she was an assistant lecturer and researcher at the University of Lille. Since October 2017, she's a researcher at SIVALab laboratory (shared laboratory between Renault/Heudiasyc/CNRS) of the Sorbonnes universités, university of Technologie of Compiègne, France. Her research is focused on the multi-sensor data fusion with fault tolerance aspect based on an informational formalism and on the integrity of localization and perception methods for autonomous vehicle.

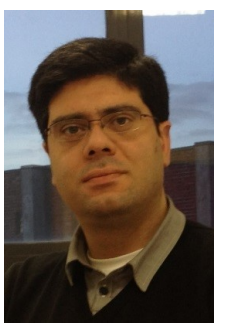

Maan El Badaoui El Najjar received the computer engineering degree and the Master of science degree from the INP Grenoble- France in control system respectively in 1999 and in 2000 . He received the $\mathrm{Ph} . \mathrm{D}$. degree in Perception and Control Systems from the University de Technology of Compiegne in 2003. In November 2011, he obtained the Habilitation à Diriger des Recherche from the University of Lille. He joined the University of Lille in 2005 as a permanent associate Professor. Since 2014, he is Full Professor at the University of Lille. He is also the head of the DiCOT Team "Diagnostic, Control and Observation for fault Tolerant Systems" of the CRIStAL Laboratory UMR CNRS 9189. His research has been focused on fault tolerant multi-sensor fusion approach which is able to effectively deal with faulty sensors of dynamic systems and has desired capacities for robotics and intelligent vehicle system applications 